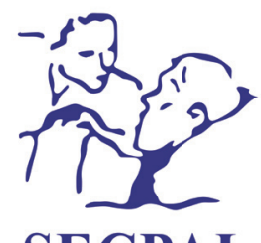

Medicina Paliativa

\title{
Conocimiento y valoración de las voluntades anticipadas del personal sanitario de unidades de hospitalización a domicilio, oncología médica, hospitales de día y servicios de urgencias
}

\author{
María Teresa García Salvador ${ }^{* 1}$, Nuria Garrido Rodríguez ${ }^{1}$, Vicente Ribes Gadea ${ }^{2}$, \\ Luis Gómez Royuela ${ }^{1}$, Silvia Forcano Sanjuan ${ }^{3}$ y Vicente Ruiz García ${ }^{4}$
}

'Unidad de Hospitalización a Domicilio, Hospital Universitario y Politécnico La Fe, Valencia, España. ${ }^{2}$ Servicio de Urgencias, Hospital La Ribera, Alzira, España. 'Unidad de Hospitalización a Domicilio, Hospital Universitario y Politécnico La Fe, Valencia, España. ${ }^{4}$ Unidad de Hospitalización a Domicilio, Hospital Universitario y Politécnico La Fe, Valencia, España

Recibido el 16 de abril de 2018

Aceptado el 11 de noviembre de 2018

\author{
PALABRAS CLAVE \\ Testamento vital, \\ instrucciones previas, \\ planificación \\ anticipada de \\ decisiones.
}

\begin{abstract}
Resumen
Objetivo: Describir el conocimiento del personal sanitario de servicios implicados en el manejo de enfermos paliativos en situación avanzada, sobre el Documento de Voluntades Anticipadas (DVA) y su Registro Nacional. Conocer cómo valoran su uso y si lo consultan habitualmente.

Material y método: Estudio multicéntrico, transversal descriptivo, mediante cuestionario anónimo cumplimentado por personal médico y de enfermería en tres centros hospitalarios. Se recogieron un total de 160 cuestionarios correspondientes a 58 médicos y 102 enfermeros, pertenecientes a Servicios de Urgencias (SU), Oncología, Unidades de Hospitalización a Domicilio $(\mathrm{HaD})$ y Hospital de Día. El cuestionario constaba de 14 preguntas tipo test de respuesta única, además de las variables demográficas y laborales pertinentes para el estudio.

Resultados: Las medias de edad de la muestra fueron de $42 \pm 9$ años, y la experiencia profesional de $17 \pm 9,3$ años. El 83,1\% de los encuestados afirmaron conocer el DVA y el 53,7\% que sabrían cumplimentarlo, pero solo un $8,1 \%$ de ellos contestaron correctamente a la pregunta de dónde localizar dicho documento. El 92,5\% de los encuestados no lo consultan nunca. Los dos encuestados que afirmaban consultarlo habitualmente, no supieron localizarlo.

Conclusiones: Existe un desconocimiento generalizado del DVA, siendo este independiente tanto de la categoría profesional como de los años de experiencia o del servicio en el que se trabaja. Incluso en los casos que dicen conocerlo, sus respuestas no corroboran tal afirmación.
\end{abstract}

*Autor para correspondencia: María Teresa García Salvador

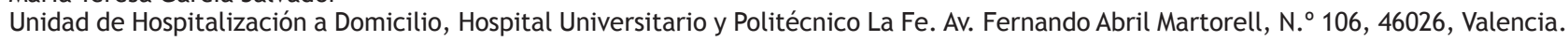

Correo electrónico: ma_garsal@hotmail.com 
KEYWORDS

Living will, advance directives, advance care planning.

\begin{abstract}
Objective: To describe the knowledge healthcare providers at the units involved in the management of advanced palliatiave care patients have of the advance directives document (ADD). To find out how do they value its use, and whether they check it out regularly.

Material and methods: A multicenter, cross-sectional, descriptive study using an anonymous questionnaire to be completed by doctors and nurses in three hospitals. A total of 160 questionnaires were collected from 58 doctors and 102 nurses working at emergency rooms (ERs), oncology departments, home hospitalization units (HHUs), and day hospital services. Questionnaires included 14 multiple choice questions with only one valid option, and also collected the demographic and labor variables required by the study.

Results: Mean age in the sample was $42 \pm 9$ years, and professional experience was $17 \pm 9.3$ years. Among responders, $83.1 \%$ claimed knowledge of the ADD, and $53.7 \%$ said they could complete it, but only $8.1 \%$ knew where to find it; $92.5 \%$ of respondents never consult it. The two respondents who claimed they usually checked it out were eventually unable to locate it.

Conclusions: Lack of knowledge about ADDs and their related National Registry is widespread, and occurs regardless of professional category or years of experience. Even in providers who claim awareness of this document the answers provided belie it.
\end{abstract}

\section{Introducción}

El Documento de Voluntades Anticipadas (DVA), también conocido como Instrucciones Previas, es el instrumento legal por el que una persona manifiesta anticipadamente su voluntad respecto a los cuidados y tratamientos que desearía recibir llegado el caso en que no sea capaz de expresarla personalmente. Se trata de una herramienta que otorga el mayor grado de autonomía al individuo. Regulada en la Ley 41/2002, de 14 de noviembre, también puede recoger deseos sobre el destino del cuerpo o de sus órganos una vez fallecido".

Sus orígenes datan de finales de los años 60 en EE. UU. ${ }^{2}$. En España, en el Convenio celebrado en Oviedo el 4 de abril de 1997, se hace referencia a que "serán tomados en consideración los deseos expresados anteriormente con respecto a una intervención médica por un paciente que, en el momento de la intervención, no se encuentre en situación de expresar su voluntad"'. Posteriormente se comenzó a legislar en las distintas comunidades autónomas, por lo que existe una gran heterogeneidad de las normas jurídicas que regulan el DVA a nivel nacional ${ }^{2}$. Con la Ley $1 / 2003$ de Derechos e Información al Paciente, y en la Comunidad Valenciana con el Decreto 168/2004, se regula el DVA y se crea el Registro Centralizado de Voluntades Anticipadas de la Comunidad Valenciana, haciendo realidad la posibilidad de ejercer uno de los derechos fundamentales del paciente, el derecho de autonomía ${ }^{4,5}$.

Tras casi veinte años de legislación, a fecha de enero de 2018, tan solo el 0,47 \% de los habitantes de la Comunidad Valenciana y el 0,53 \% de los españoles tenían registradas las Instrucciones Previas ${ }^{6}$. Teniendo en cuenta que el envejecimiento de la población y la cronicidad de las enfermedades obliga al planteamiento de estrategias sanitarias que potencien el autocuidado y el empoderamiento de las personas con respecto a las decisiones sobre su salud, este escaso registro es sorprendente. Esto podría deberse, según apuntan algunos autores, a que las personas mayores prefieren no pensar en la muerte y a factores religiosos y de nivel cultural ${ }^{7,8}$. La ausencia de registros conlleva que ante situaciones de enfermedad grave o de final de vida sea el personal sanitario y los familiares los que tomen decisiones independientemente de la voluntad del paciente.

Con el impulso actual hacia unos cuidados paliativos de calidad y la promoción de la muerte digna, resulta necesario el diálogo entre paciente y profesionales para explorar los deseos de este y avanzar en la planificación de decisiones, siendo imprescindible crear un clima de confianza con los profesionales que atienden habitualmente al paciente 9,10 . Pero en ocasiones, una situación de gravedad o urgencia puede provocar que sea otro equipo el que atienda al paciente, de ahí la importancia que cobra la existencia de un registro de las voluntades accesible y operativo ${ }^{11}$. En los servicios de urgencias a menudo se producen situaciones en las que es necesaria la toma de decisiones de una forma inmediata en pacientes graves, con facultades mermadas o sin acceso a familiares, teniendo que asumir la responsabilidad de decidir, entre otras cosas, si instaurar maniobras de reanimación en una persona a la que se desconoce por completo. Un DVA podría ser de gran ayuda para orientar el tipo de intervención sanitaria más adecuada a realizar.

En estudios previos, la mayoría del personal sanitario se quejaba de la escasa información que tenían sobre el DVA. Esa falta de información era mayor aún en los pacientes, que llegaban a relacionarlo con la eutanasia o la donación de órganos ${ }^{12-16}$. Sin embargo, la actitud hacia el DVA, tanto de profesionales como de pacientes, es muy favorable ${ }^{15}$. 
Finalmente, es necesario recordar que el DVA y su registro son tan solo una herramienta, ya que lo fundamental es promover la reflexión comunitaria y fomentar las conversaciones sobre el final de la vida.

Por todo ello, el objetivo de este estudio es explorar los conocimientos y actitudes que tiene el personal sanitario de servicios implicados en la atención a pacientes en situación crónica o de enfermedad avanzada, en aras de promover el uso del DVA en la ciudadanía asumiendo que deben ser estos profesionales los mayores conocedores de su existencia.

\section{Material y métodos}

Se realizó un estudio observacional transversal, consistente en la evaluación de cuestionarios anónimos autocumplimentados por personal médico y de enfermería perteneciente a los Servicios de Urgencias (SU), Oncología (SO), Hospital de día (HD) y Unidad de Hospitalización a Domicilio $(\mathrm{HaD})$ de tres centros hospitalarios de la provincia de Valencia. Dichos profesionales aceptaron voluntariamente participar en el estudio. Se escogieron estos servicios hospitalarios por su mayor relación con el paciente en situación de final de vida. La recogida de cuestionarios se realizó durante un trimestre del 2017. Se excluyeron estudiantes y residentes.

Los cuestionarios se remitieron a los jefes de servicio y supervisores mediante un enlace a la aplicación online Google Forms. Dado que desconocíamos a cuántos profesionales se remitió dicho enlace, calculamos nuestro tamaño muestral en base a una población desconocida, calculando un nivel de prevalencia del $10 \%$ (cifra extraída de los estudios de Bachiller ${ }^{14}$ y Santos de Unamuno ${ }^{7}$, para el porcentaje de profesionales sanitarios conocedores del DVA) con una fiabilidad del $95 \%$. Se obtuvo un tamaño muestral de 139 individuos.

El cuestionario, diseñado ad hoc según la bibliografía consultada ${ }^{15,17}$ y validado online por un grupo de expertos, incluía variables demográficas (edad, sexo, hospital y servicio de trabajo, años de experiencia y categoría profesional) que permitían definir la muestra de estudio. Posteriormente se solicitaba cumplimentar 14 preguntas, de respuesta cerrada (tipo test de respuesta única). En dos ítems se solicitaba la justificación o ampliación de la respuesta.

Las preguntas que conforman el cuestionario se pueden ver en la Tabla I.

Se incluyó una pregunta control: ¿sabría dónde y cómo localizar el DVA de los pacientes a su cargo?, para corroborar el conocimiento real de los encuestados sobre el documento.

Las variables se expresaron como media \pm desviación estándar si eran cuantitativas y en porcentajes si eran cualitativas. La comparación entre grupos se realizó mediante la prueba de Chi cuadrado, alternativamente, mediante la prueba exacta de Fisher cuando los efectivos calculados fueron inferiores a 5 y se calculó la razón de Odds con un intervalo de confianza del $95 \%$. Se consideraron estadísticamente significativas aquellas variables con valor de $p<0,05$.

La realización del proyecto fue aprobada por los Comités Éticos de Investigación del hospital La Fe y del hospital La Ribera. En los cuestionarios se incluía un consentimiento informado en el que el que se indicaba la confidencialidad y la autorización a los investigadores a utilizar estos datos exclusivamente para el estudio.

\section{Resultados}

Se recibieron un total de 160 cuestionarios. La edad media fue de $42,24 \pm 9,12$ y el porcentaje de mujeres del 73,8 \%. El 63,8 \% de los cuestionarios pertenecían a enfermería y el resto a médicos. Respecto a la distribución por servicios un $40 \%$ correspondía al SU, un $25 \%$ a HaD, un $20 \%$ al SO y un $15 \%$ a HD.

La experiencia profesional media fue de $16,86 \pm 9,3$. Los profesionales del SO son los que representaban menos años de experiencia profesional (14 años), mientras que en los otros tres servicios oscilaba entre 17 y 20 años. La diferencia entre categorías profesionales era apenas apreciable, con una media de 16 años para enfermería y de 17 años para el personal médico.

El 83,13\% de la muestra obtenida manifestaba conocer el DVA, siendo este dato independiente del grupo de edad al que pertenecen, sexo, años de experiencia laboral, categoría profesional y servicio hospitalario.

El 46,3\% de los encuestados desconocía cómo se formaliza legalmente un DVA, independientemente del sexo, grupo de edad o años de experiencia. Sin embargo, existía una relación estadísticamente significativa entre el conocimiento de cómo formalizar el documento y el servicio de trabajo ( $p=0,012$ ). Solo un $29,2 \%$ del personal de HD manifestaba conocer el procedimiento, frente al 67,2 \% de los SU. Dicho conocimiento es mayoritario entre el personal médico, con una relación estadísticamente significativa $(p=0,01)$. También se encontró relación estadísticamente significativa con los años de experiencia profesional $(p=0,03)$. Los facultativos con experiencia profesional inferior a 5 años desconocían en su mayoría el procedimiento $(p=0,006)$. Analizando la relación entre servicios y categoría profesional se halló relación estadística para enfermería $(p=0,046)$ y médicos $(p=0,041)$. Las enfermeras de Oncología y Urgencias eran las que, en mayor número, decían conocer el procedimiento (60\%), en cuanto a los médicos, los oncólogos eran los que en menor medida manifestaron el saber cómo realizarlo $(38,5 \%)$, en comparación con sus compañeros de otros servicios (74,2 \% médicos de SU y 78,6 \% médicos de $\mathrm{HaD}$ ).

A la pregunta de si conocían el Registro Nacional de Voluntades Anticipadas, un 56,3\% respondió afirmativamente. No se encontró relación significativa entre el conocimiento del Registro Nacional de Voluntades Anticipadas y los años de experiencia profesional, sexo ni servicio de trabajo. Tampoco la hubo comparándolo con la edad, pero los datos reflejaron que el 100 \% de los mayores de 60 años afirmaban conocerlo. Sí se obtuvo relación con la categoría profesional, ya que los médicos manifestaron conocer en mayor porcentaje que Enfermería dicho Registro ( $p=0,002)$. Nuevamente son los oncólogos los facultativos que expresaron menor conocimiento de su existencia (38,5 \% vs. $80,6 \%$ SU, 85,7 \% $\mathrm{HaD})(\mathrm{p}=0,007)$.

Un $28 \%$ de los encuestados respondió que sabría dónde localizar el DVA de sus pacientes. Sin embargo, únicamente el $8,1 \%$ (13 participantes) respondió correctamente la pregunta sobre la localización del DVA dentro de la Historia Clínica de Atención Primaria. Este conocimiento mostraba una relación estadísticamente significativa $(p<0,05)$ con la categoría profesional, ya que solo un cuestionario correspondía a Enfermería y el resto a personal médico. 


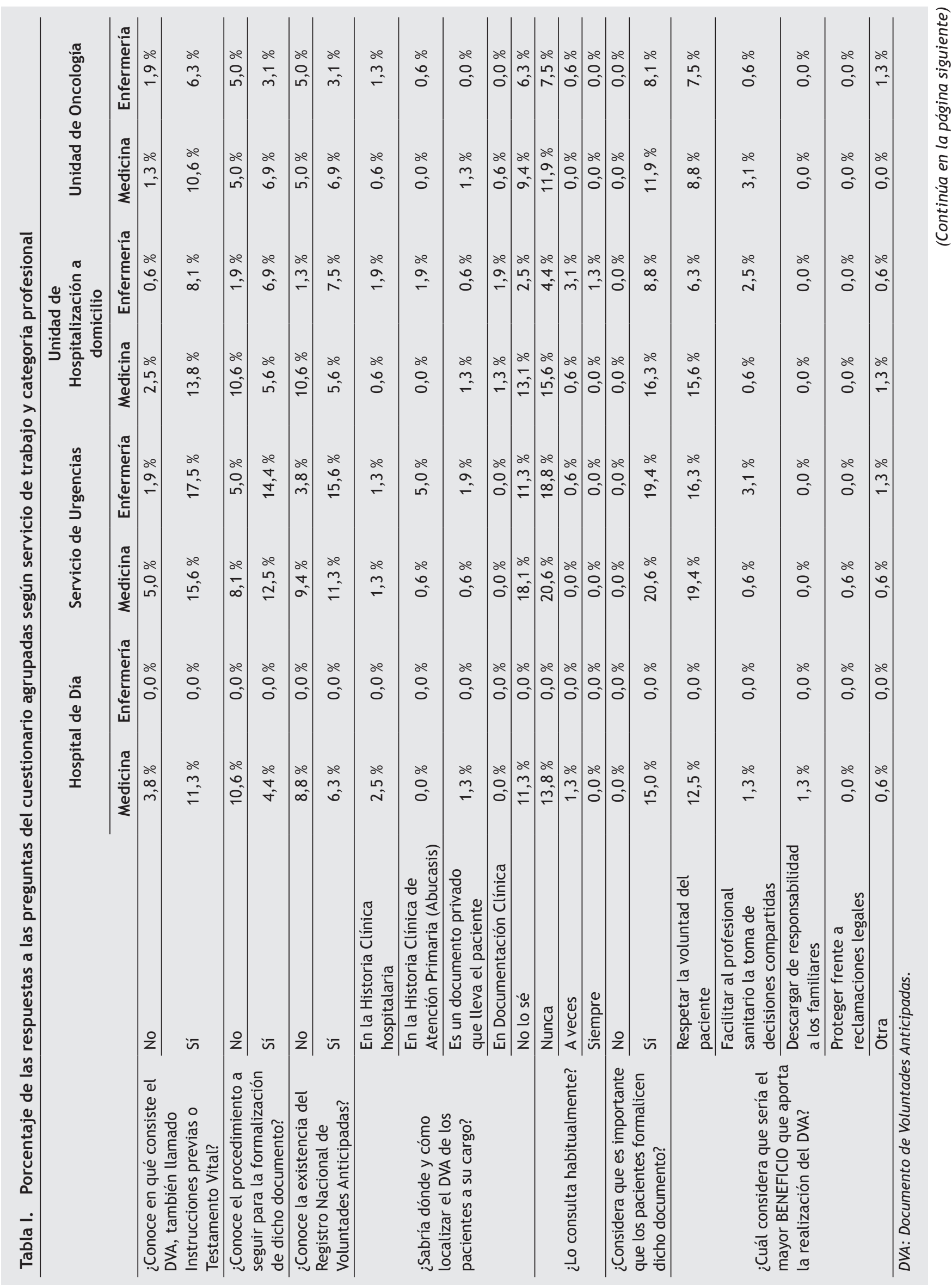




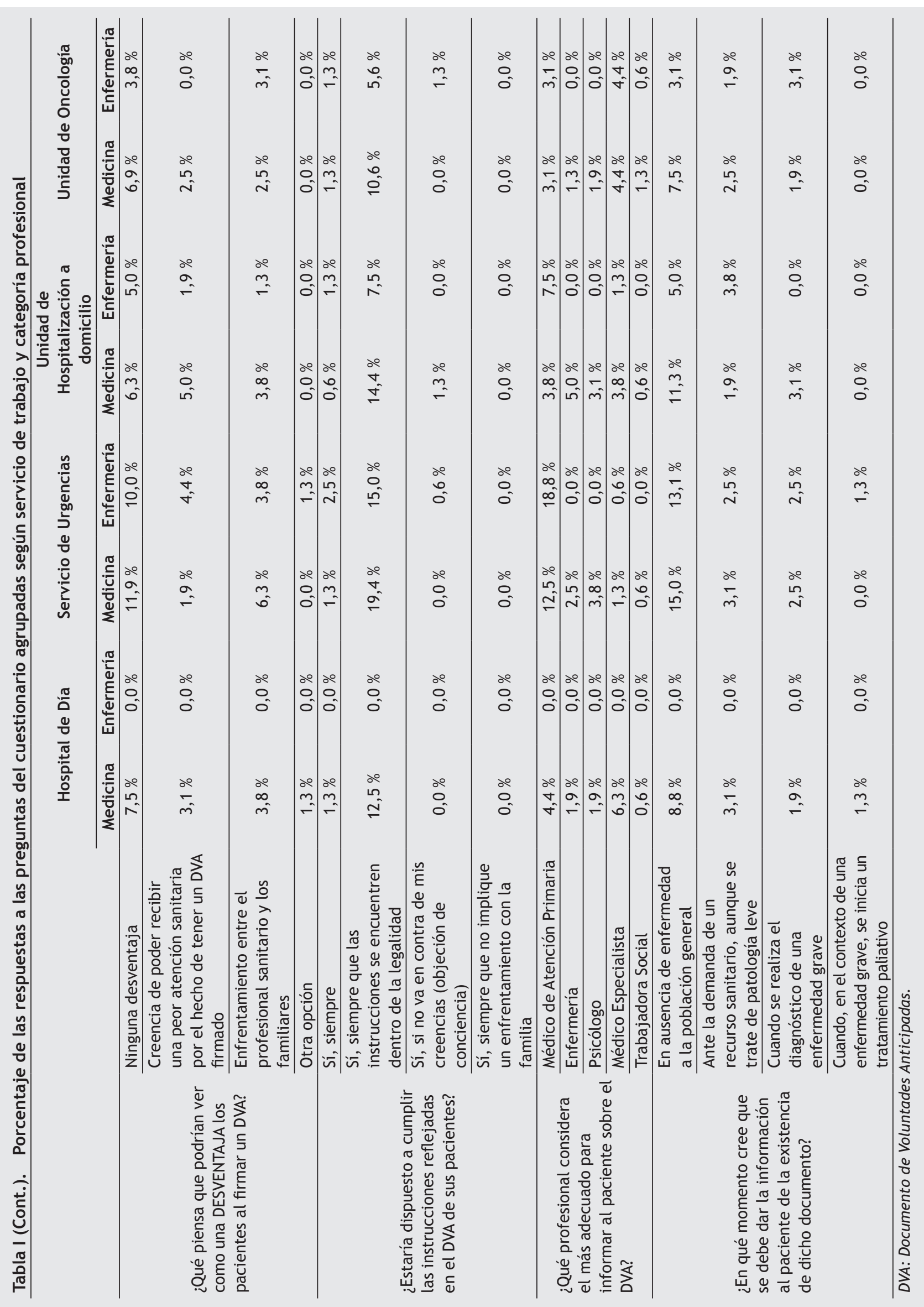




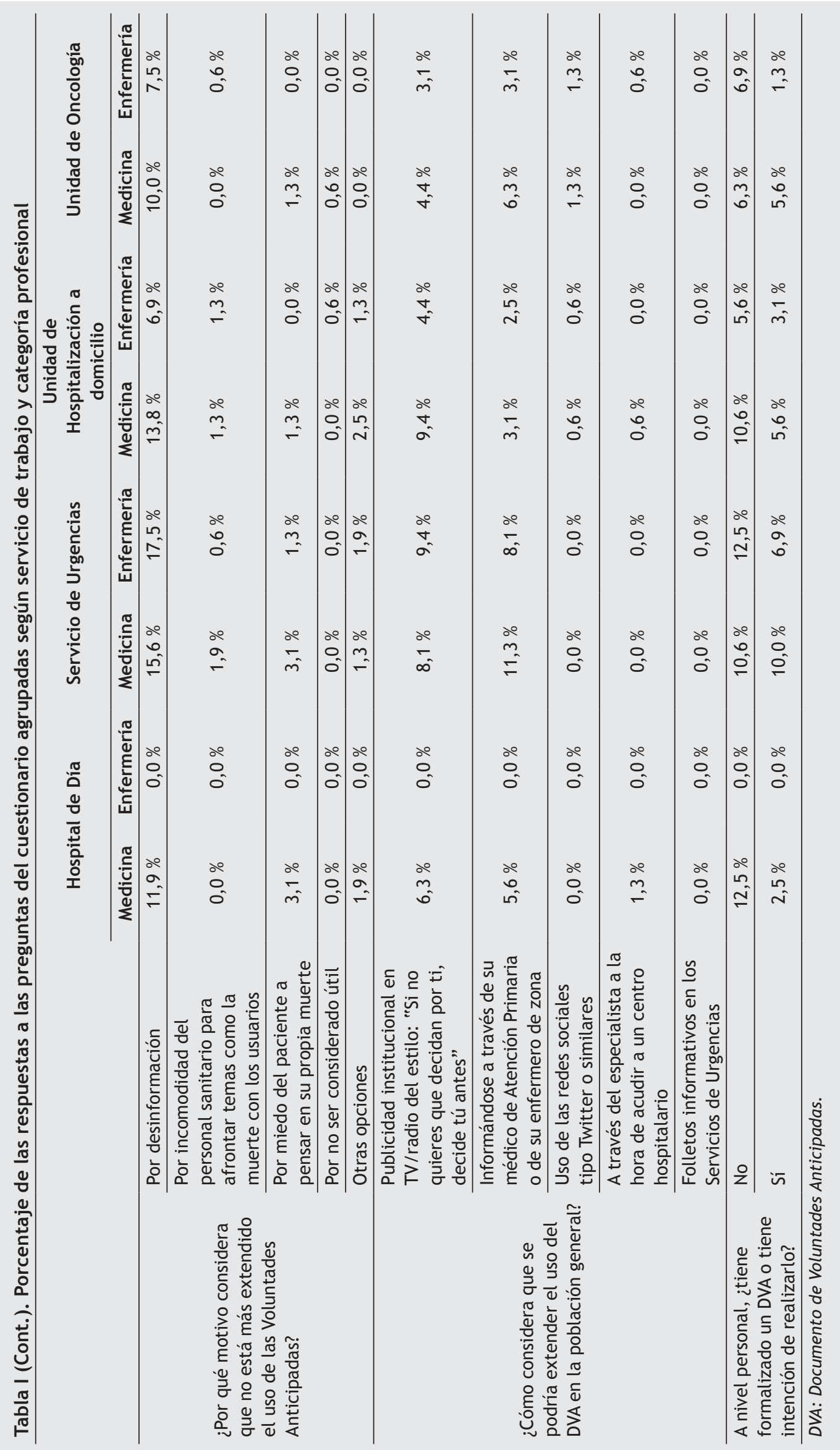


Destaca el conocimiento de dónde localizar el DVA de los médicos de Urgencias $(25,8 \%)$ y de $\mathrm{HaD}(21,4 \%)$, frente a las enfermeras de sus respectivos servicios ( $3 \%$ y $0 \%$ ), siendo en ambos casos estadísticamente significativo $(p=0,03$ y $p=0,008$, respectivamente). En cambio, en la Unidad de Oncología este ítem se respondió de forma similar entre ambas categorías profesionales.

De los 160 encuestados, solo dos respondieron que consultaban siempre el DVA de sus pacientes, mientras que 10 manifestaron que lo consultaban ocasionalmente. El resto reconoció que nunca lo habían consultado. Esos dos cuestionarios pertenecían a médicos de $\operatorname{HaD}(p=0,022)$ y, aunque decían consultarlo siempre, respondieron de forma errónea a la pregunta de dónde se localiza el DVA. De los 10 que respondieron que lo consultaban ocasionalmente, solo tres encuestados sabrían localizarlo adecuadamente (Figura 1).

Todos los encuestados señalaron la importancia de la cumplimentación del DVA. Como mayor beneficio para su realización, el $86,3 \%$ de la muestra resaltó el respeto a la voluntad del paciente.

El 51,25\% de los encuestados manifestó no encontrar ninguna desventaja a tener cumplimentado un DVA. Entre el resto, la desventaja más destacada fue el posible conflicto entre el personal sanitario y las familias $(24,6 \%)$, seguida por el miedo del paciente a recibir una peor asistencia sanitaria (18,8\%). En la opción de respuesta libre algunas de las contestaciones fueron el miedo a cargar de responsabilidad al representante familiar y la dificultad de modificación con el cambio de opinión del paciente.

El $85 \%$ de los encuestados respondió que cumpliría las instrucciones incluidas en el DVA, siempre que estas estuvieran dentro de la legalidad. El 9,4\% lo cumpliría en cualquier condición, y un 3,1 \% lo cumpliría únicamente si fuera compatible con sus creencias o valores. No se encontró relación estadística entre las respuestas a esta pregunta y la edad o los años de experiencia profesional.

Más de la mitad de los encuestados $(53,1 \%)$ consideraron que el profesional más adecuado para informar sobre el DVA es el médico de Atención Primaria, seguido por el médico especialista $(21,88 \%)$. Se encontró relación estadísticamente significativa $(\mathrm{p}<0,001)$ con la categoría profesional, ya que la gran mayoría de los médicos respondieron que el

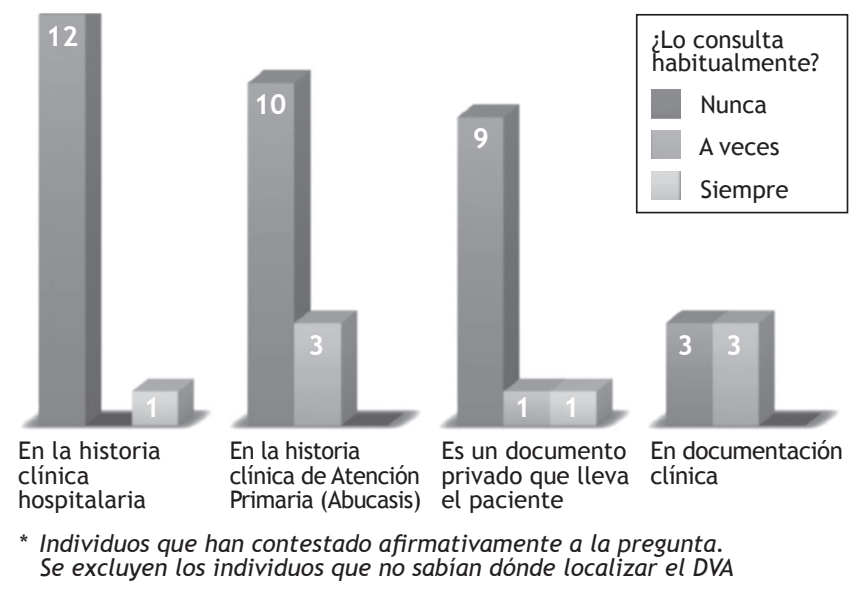

Figura 1. ¿Sabría cómo y dónde localizar el DVA de sus pacientes? más indicado para informar sobre el DVA es el médico de Atención Primaria, a excepción de un 6,3\% que cree como mejor responsable al médico especialista. En cambio, un 23,8 \% de enfermeras consideraron que debe ser el médico de Atención Primaria quien informe sobre el DVA.

Se encontró también relación estadísticamente significativa con el servicio de trabajo ( $p<0,001)$, ya que tanto el SO como el HD se decantaron por el médico especialista, mientras que los SU y Unidades de HaD lo hicieron por el médico de Atención Primaria. En ningún servicio los médicos incluyeron a la enfermería como personal responsable de esta tarea, sin embargo, las enfermeras de HaD consideraron que ellas son las profesionales más indicadas para informar de la formalización de este documento.

El 63,75\% creyó que el mejor momento de informar de la existencia del DVA es en ausencia de enfermedad.

El 83,13\% de los profesionales encuestados consideró que su uso no está más extendido por desinformación, frente a un $10 \%$ que lo achacaron al miedo del paciente a pensar en su muerte, y un 5,63\% responsabiliza a la incomodidad del personal sanitario a afrontar con los usuarios temas como la muerte. Un 1,25 \% no lo considera útil.

En cuanto a la pregunta de cuál consideraría la mejor opción para extender su uso, encontramos que un $45 \%$ consideró que debe hacerse por medio de publicidad institucional a través de la radio o televisión, seguido de un $40 \%$ partidario de aumentar la información a través de su médico o enfermera de Atención Primaria. El resto de las alternativas de difusión como el uso de redes sociales, la información por parte del médico especialista o combinar medios de comunicación para adecuar la información al rango de edad del usuario fueron menos significativas para los encuestados.

Se encontró relación estadísticamente significativa entre el método de elección para la divulgación de DVA y la variable del servicio de trabajo $(p=0,011)$. HaD se decantaba por aumentar la publicidad institucional, mientras que urgencias y oncología lo hicieron por el médico o enfermera de Atención Primaria. Ninguno de los encuestados se decantó por la propuesta de divulgación en los servicios de urgencias a través de folletos informativos.

El $65 \%$ de los encuestados manifestó no tener realizado un DVA ni tener como objetivo próximo el formalizar uno. Aunque no se encontró relación estadística, se observa que tanto los oncólogos como las enfermeras de HD fueron los menos interesados en realizarlo con solo un 15 y un $16 \%$, respectivamente, a diferencia del resto del personal encuestado que ronda una media del $35 \%$.

\section{Discusión}

A pesar de que todos los encuestados consideraban el DVA como un instrumento útil para la mejora de la calidad en la atención a los pacientes, los resultados muestran el escaso conocimiento que se tiene de él. Estos datos concuerdan con la mayoría de la bibliografía consultada ${ }^{12-16}$. En el estudio de Champer Blasco y cols., que valora los conocimientos y las actitudes de los profesionales sanitarios de los equipos de Atención Primaria sobre el DVA, se concluye la necesidad de actividades dirigidas a mejorar el conocimiento de los aspectos legales, de registro y consulta de dicho documento ${ }^{18}$. En un estudio realizado a profesionales médicos y 
de enfermería del SUMMA 112 solo el $18 \%$ afirmó conocer los detalles de la legislación actual sobre el documento de voluntades anticipadas ${ }^{19}$.

Los profesionales que más conocen el procedimiento del DVA son los médicos de los Servicios de Urgencias, seguidos de los de HaD, y la enfermería de Oncología, y estos tres grupos son los de mayor edad de la muestra. Sin embargo, los profesionales menos informados sobre este documento son los Oncólogos que, además, son los más jóvenes de nuestra muestra de estudio. Esto podría ser debido a que en el momento en que se instauró el uso del DVA en la Comunidad Valenciana fue divulgado a nivel general a través de la elaboración de una guía informativa en el año 2005, sin que posteriormente se mantuviera el impulso inicial. Sin embargo, no se ha encontrado bibliografía con la que poder comparar este resultado. En general, los datos del estudio revelan que existe un mayor conocimiento de esta herramienta por parte del personal médico en comparación con el personal de enfermería, dato que coincide con el obtenido por Toro Flores ${ }^{13}$. Una de las posibles causas de este hecho podría ser la falta de accesibilidad al DVA por parte de la enfermería hospitalaria, hecho este que creemos debería ser modificado. Además, Antolín y cols. concluyen en su trabajo que es enfermería la que "puede realizar una labor clave en la difusión de esta información, ya que su permanente contacto con el enfermo y su familia facilita el inicio del diálogo acerca de los aspectos de autonomía y toma de decisiones" ${ }^{\prime 2}$. Varios estudios sobre el conocimiento del DVA por parte del personal de enfermería podrían ir en este sentido ${ }^{21,22}$.

Recientemente se están instaurando alarmas en la historia clínica informática hospitalaria que avisan de que el paciente tiene formalizado un DVA, aunque no permite su acceso directo al contenido. Esta dificultad para el acceso a visualizar el documento podría motivar la escasa consulta que de él se hace, tal y como revela el bajo porcentaje de profesionales que dice consultarlo habitualmente $(7,5 \%)$. Y, a pesar de afirmar consultarlo, solo un $25 \%$ sabría localizarlo adecuadamente.

En un estudio realizado a pacientes crónicos que acudían a los servicios de urgencias por descompensaciones de su enfermedad se concluyó que solo un $19 \%$ de los pacientes tenía conocimiento acerca del DVA, y apenas un $3 \%$ había recibido esa información de su médico habitual ${ }^{19}$. Estos escasos porcentajes pueden estar relacionados con los datos obtenidos en la cuestión de cuál es el mejor momento para informar sobre la existencia del DVA, ya que un significativo $63,75 \%$ contestó que lo idóneo es informar en ausencia de enfermedad. Fueron los profesionales más veteranos y con mayor experiencia los que más se decantaron por no esperar a informar sobre dicho documento en caso de enfermedad avanzada o con tratamiento paliativo establecido.

Dado que la muestra estudiada pertenecía a personal implicado en situaciones de últimos días del paciente, pensamos que si el estudio se ampliara a otros profesionales no implicados en este campo, el desconocimiento del DVA sería aún mayor.

Por otra parte, por las respuestas obtenidas parece que un gran porcentaje de los encuestados hubieran confundido el DVA con la tarjeta de donante de órganos, al afirmar que se trataba de un documento que lleva el paciente.

Finalmente, un dato que nos ha sorprendido es que, a pesar de su reconocida utilidad para el paciente y el equi- po sanitario, de la predisposición de los profesionales a la difusión del DVA y al respeto a los deseos reflejados en él, siempre que se encuentren dentro de la legalidad, se haya detectado una escasa motivación para formalizar unas instrucciones previas propias entre los médicos y enfermeras encuestados ${ }^{23}$. En consecuencia, consideramos que el trabajo de formación e información, junto con la ruptura de los tabúes que dificultan tratar abiertamente el tema de una muerte digna conforme a los deseos expresados por la persona, debe comenzar desde dentro del ámbito sanitario ${ }^{24}$.

\section{Agradecimientos}

Queríamos agradecer toda la ayuda prestada por Eva Rueda, Supervisora de Investigación de la Subdirección de Enfermería del Hospital La Fe, desde el inicio del proyecto.

\section{Conflicto de intereses}

Todos los autores declaran la no existencia de conflictos de intereses.

\section{Financiación}

Este artículo no ha recibido ningún tipo de financiación.

\section{Bibliografía}

1. Ley $41 / 2002$, de 14 de noviembre, Básica Reguladora de la Autonomía del Paciente y de Derechos y Obligaciones en Materia de Información y Documentación Clínica. Disponible en: https: / / www. boe.es/boe/dias/2002/11/15/pdfs/A4012640132.pdf

2. Seoane JA. Derecho y planificación anticipada de la atención: panorama jurídico de las instrucciones previas en España. DS. 2006;14:285-96.

3. Instrumento de Ratificación del Convenio para la protección de los derechos humanos y la dignidad del ser humano con respecto a las aplicaciones de la Biología y la Medicina (Convenio relativo a los derechos humanos y la biomedicina). Publicado en: BOE núm. 251, de 20 de octubre de 1999, páginas 36825 a 36830 .

4. Decreto 168/2004, de 10 de septiembre, del Consell de la Generalitat, por el que se regula el Documento de Voluntades Anticipadas y se crea el Registro Centralizado de Voluntades Anticipadas de la Comunidad Valenciana. [2004/9560]. Disponible en: http: // www.dogv.gva.es/datos/2004/09/21/pdf/2004_9560.pdf.

5. Guía de las Voluntades Anticipadas. Valencia: Conselleria de Sanitat ${ }^{\circ}$; 2010. Disponible en: http://www.san.gva.es/docum ents/151744/192114/V.753-2010.pdf

6. Registro Nacional de Instrucciones Previas. Disponible en: https: / / www.msssi.gob.es/ciudadanos/rnip/home.htm.

7. Santos de Unamuno C. Documento de voluntades anticipadas: actitud de los pacientes de atención primaria. Aten Primaria. 2003;32:1-8.

8. Andrés-Pretel F, Navarro Bravo B, Párraga Martínez I, de la Torre García MA, Jiménez Del Val MD, López Torres Hidalgo J. Conocimientos y actitudes de los mayores hacia el documento de voluntades anticipadas. Gac Sanit. 2012;26:570-3. 
9. Simón Lorda P, Barrio Cantalejo IM, Alarcos Martínez FJ, Barbero Gutiérrez J, Couceiro A, Hernando Robles P. Ética y muerte digna: propuesta de consenso sobre un uso correcto de las palabras. Rev Calidad Asistencial. 2008;23:271-85.

10. Borasio GD. Sobre el buen morir. Barcelona: Ed. Plataforma; 2014.

11. Altisent R. Planificación anticipada de la asistencia. Se necesita una revolución educativa. Aten Primaria. 2013;45:402-3.

12. Navarro Bravo B, Sanchez García M, Andrés Pretel F, Juárez Casalengua I, Cerdá Díaz R, Párraga Martínez I, et al. Declaración de voluntades anticipadas: estudio cualitativo en personas mayores y médicos de Atención Primaria. Aten Primaria. 2011;43:11-7.

13. Toro Flores R, Silva Mato A, Piga Rivero A, Alfonso Galán MT. Conocimientos y actitudes de médicos y enfermeras sobre las instrucciones previas. Aten Primaria. 2013;45:404-8.

14. Bachiller Baeza A, Hernández de Miguel S, Martínez Queipo M, Delgado Martínez R, Domínguez Cano V. Testamento vital: la opinión médica en la provincia de Valladolid. Met Enferm. 2004;7:24-7.

15. Contreras-Fernández E, Rivas-Ruiz F, Castilla-Soto J, MéndezMartínez C. Conocimientos y actitudes de los profesionales sanitarios en el proceso de declaración de las voluntades vitales anticipadas. Aten Primaria. 2015;47:514-22.

16. Simón Lorda P, Tamayo Velázquez MI, Vázquez Vicente A, Durán Hoyos A, Pena González J, Jiménez Zurita P. Conocimientos y actitudes de los médicos de dos áreas sanitarias sobre las voluntades vitales anticipadas. Aten Primaria. 2008;40:61-8.

17. Contreras Fernández E, Barón López FJ, Méndez Martínez C, Canca Sánchez JC, Cabezón Rodríguez I, Rivas Ruiz F. Validación del cuestionario de conocimientos y actitudes de los profesionales sanitarios en el proceso de declaración de voluntades vitales anticipadas. Aten Primaria. 2017;49:233-9.
18. Champer Blasco A, Caritg Monfort F, Marquet Palomer R. Conocimientos y actitudes de los profesionales de los equipos de atención primaria sobre el documento de voluntades anticipadas. Aten Primaria. 2010;42:463-9.

19. Mateos Rodríguez A, Huerta Arroyo A, Benito Vellisca MA. Instrucciones previas: actitud de los profesionales de emergencias. Emergencias. 2007;19:241-4.

20. Antolín A, Ambrós A, Mangirón P, Alves D, Sánchez M, Miró 0 . Conocimientos acerca de su enfermedad y de los documentos de voluntades anticipadas en los pacientes con enfermedades crónicas evolutivas descompensadas que consultan en urgencias. Emergencias. 2007;19:245-50.

21. Simón Lorda P, Tamayo Velázquez MI, González Rubio MJ, Ruiz Díaz P, Moreno González J, Rodríguez González MC. Conocimientos y actitudes del personal de enfermería acerca de las voluntades anticipadas en 2 áreas sanitarias de Andalucía. Enferm Clin. 2008;18:11-7.

22. Álvarez Trabanco S, Argüelles Otero L, Álvarez Fernández M, Fernández Prieto T, Blanco Gutiérrez ML, Del Castillo Arévalo F. Conocimientos y actitud de las enfermeras de Atención Primaria sobre las Voluntades Anticipadas. RqR Enfermería Comunitaria (Revista SEAPA). 2015;3:66-77.

23. Aguilar-Sánchez JM, Cabañero-Martínez MJ, Puerta Fernández F, Ladios-Martín M, Fernández-de-Maya J, Cabrero-García J. Grado de conocimiento y actitudes de los profesionales sanitarios sobre el documento de voluntades anticipadas. Gac Sanit. 2018;32:339-45.

24. Nebot C, Ortega B, Mira JJ, Ortiz L. Morir con dignidad. Estudio sobre voluntades anticipadas. Gac Sanit. 2010;24:437-45. 